\title{
CARACTERIZACIÓN DEL CONTENIDO POLÍNICO INVERNAL DE LA ATMÓSFERA DE MÁLAGA
}

\author{
Marta RECIO, M. Mar TRIGO, F. Javier TORO y Baltasar CABEZUDO
}

\begin{abstract}
RESUMEN. Caracterización del contenido polínico invernal de la atmósfera de Málaga. En el presente trabajo se realiza un estudio del contenido polínico de la atmósfera de Málaga durante el periodo invernal (Enero-Marzo) tomando los registros obtenidos en los años 1992-96. En el espectro polínico se encuentran representados 31 tipos polínicos, de los cuáles se estudian tan sólo 12, ya que el resto son pólenes de reflotación o de taxones típicamente primaverales que en Málaga empiezan a aparecer durante el mes de Marzo. La mayor parte del polen detectado corresponde a Cupressaceae, seguido de Pinaceae, Urticaceae y Platanus, responsables de que las mayores concentraciones se produzcan durante los meses de Febrero y Marzo, mientras que en Enero los niveles de polen permanecen muy bajos. También aparecen taxones típicamente invernales como Alnus, Ulmus, Populus y Salix, aunque en concentraciones muy bajas debido a que las grandes poblaciones de estas especies se encuentran muy alejadas del punto de muestreo.
\end{abstract}

Palabras clave. Aerobiología, polen, invierno, Málaga, sur de España.

ABSTRACT.Characterization of winter pollen content in the atmosphere of Malaga. In this work, the winter pollen content of the atmosphere of Malaga is studied for the period 1992-96. 31 pollen types are represented in the pollen spectrum, but only 12 are studied because the rest are reflotating or typically spring pollens, which start their pollination period in early March. Most of the pollen detected corresponds to Cupressaceae, followed by Pinaceae, Urticaceae and Platanus. These are responsible of the high concentrations registered during February and March, while in January the total pollen levels are lower. Other typically winter pollen types, such as Alnus, Ulmus, Populus and Salix, appear in low concentrations, since their originating populations are situated quite far from the sampling site.

Key words. Aerobiology, pollen, winter, Malaga, southern Spain.

\section{INTRODUCCIÓN}

Las cantidades de polen, así como la composición del espectro polínico que se detectan en la atmósfera a lo largo del año varían considerablemente, contrastando los periodos con concentraciones muy elevadas de polen con otros en los que dichas concentraciones son prácticamente nulas.
En el Sur de Europa, es durante la época primaveral cuando se detectan los niveles más elevados de polen aerovagante, especialmente en los meses de Abril y Mayo, época en la que se centra una buena parte de los estudios aerobiológicos. Sin embargo, en latitudes más meridionales (costa mediterránea) también se detectan concentraciones importantes de polen durante el invierno y el espectro polínico 
invernal puede ser tan rico en especies como el primaveral aunque, las bajas concentraciones que presentan muchos de los tipos polínicos detectados sea la causa de que habitualmente su presencia no sea puesta de manifiesto.

En el presente trabajo se pretende realizar un estudio detallado del espectro polínico que aparece en la atmósfera de la ciudad de Málaga durante los meses de invierno, época en que aumenta considerablemente el número de personas residentes en la Costa del Sol procedente de otros países más septentrionales, y en donde muchos de los pólenes aquî estudiados (Alnus, Ulmus, Populus y Salix) aparecen en concentraciones extremadamente altas, produciendo en esas zonas trastornos alérgicos a un porcentaje importante de la población (Spieksma, 1991; Spieksma \& Frenguelli, 1991; Nilsson \& Spieksma, 1992).

\section{MATERIAL Y MÉTODOS}

El muestreo se ha realizado por medio de un captador volumétrico Burkard situado sobre la azotea de la Facultad de Medicina, Campus de Teatinos, a unos $15 \mathrm{~m}$ del nivel del suelo. Los recuentos polínicos se han realizado siguiendo la metodología recomendada por la Red Española de Aerobiología, REA (Domínguez et al., 1991).

Las gráficas de concentraciones polínicas se han elaborado utilizando medias móviles de cinco días, tomándose como datos básicos los valores medios diarios de los 5 años a los que

\begin{tabular}{llll}
\hline Invierno & Primavera & Verano & Otoño \\
\hline 7383 & 17104 & 1754 & 1478 \\
$26,63 \%$ & $61,71 \%$ & $6,33 \%$ & $5,33 \%$ \\
\hline
\end{tabular}

Tabla 1. Valores totales y porcentajes con respecto al total anual registrados durante cada una de las estaciones del año por los pólenes totales (media de 5 años). Total and percentage values of the total pollen counts during each season of the year (5 years average). corresponde el período de muestreo (199296). Las escalas se han adaptado a los valores máximos alcanzados por cada tipo polínico.

\section{RESULTADOS Y DISCUSIÓN}

Durante los meses de Enero a Marzo del periodo estudiado se han detectado aproximadamente una cuarta parte del polen total anual (tab. 1), correspondientes a 31 tipos polínicos que aparecen de una manera regular en la atmósfera de Málaga. De estos 31 tipos polínicos sólo 10 son típicamente invernales (Cupressaceae, Pinaceae, Platanus, Mercurialis, Populus, Artemisia, Alnus, Ulmus, Fraxinus y Salix) y, por tanto, objeto de estudio detallado en el presente trabajo. Junto con éstos tipos polínicos, se estudian otros dos (Urticaceae y Gramineae), que aunque aparecen a lo largo de todo el año, alcanzan niveles apreciables durante el período invernal. El resto de los tipos polínicos aparecen por reflotación o por tratarse de especies típicamente primaverales que inician su periodo de polinación durante el mes de Marzo, debido a la benignidad del clima de Málaga, como Olea europaea, ChenopodiaceaeAmaranthaceae, Plantago, Quercus o Rumex. Por último, otros pólenes, como Ricinus y Asteraceae aparecen esporádicamente, y en concentraciones bajas en cualquier época del año.

En la figura 1 se muestra la evolución media que han presentado las concentraciones atmósféricas de estos doce tipos polínicos estudiados durante los cinco años de muestreo (1992-96). Se observa que Cupressaceae, Pinaceae, Urticaceae y Platanus alcanzan mayores concentraciones, mientras que Mercurialis, Gramineae, Populus y Artemisia aparecen en concentraciones más bajas, y Alnus, Ulmus, Fraxinus y Salix en concentraciones muy bajas.

Durante el mes de Enero las cantidades de 

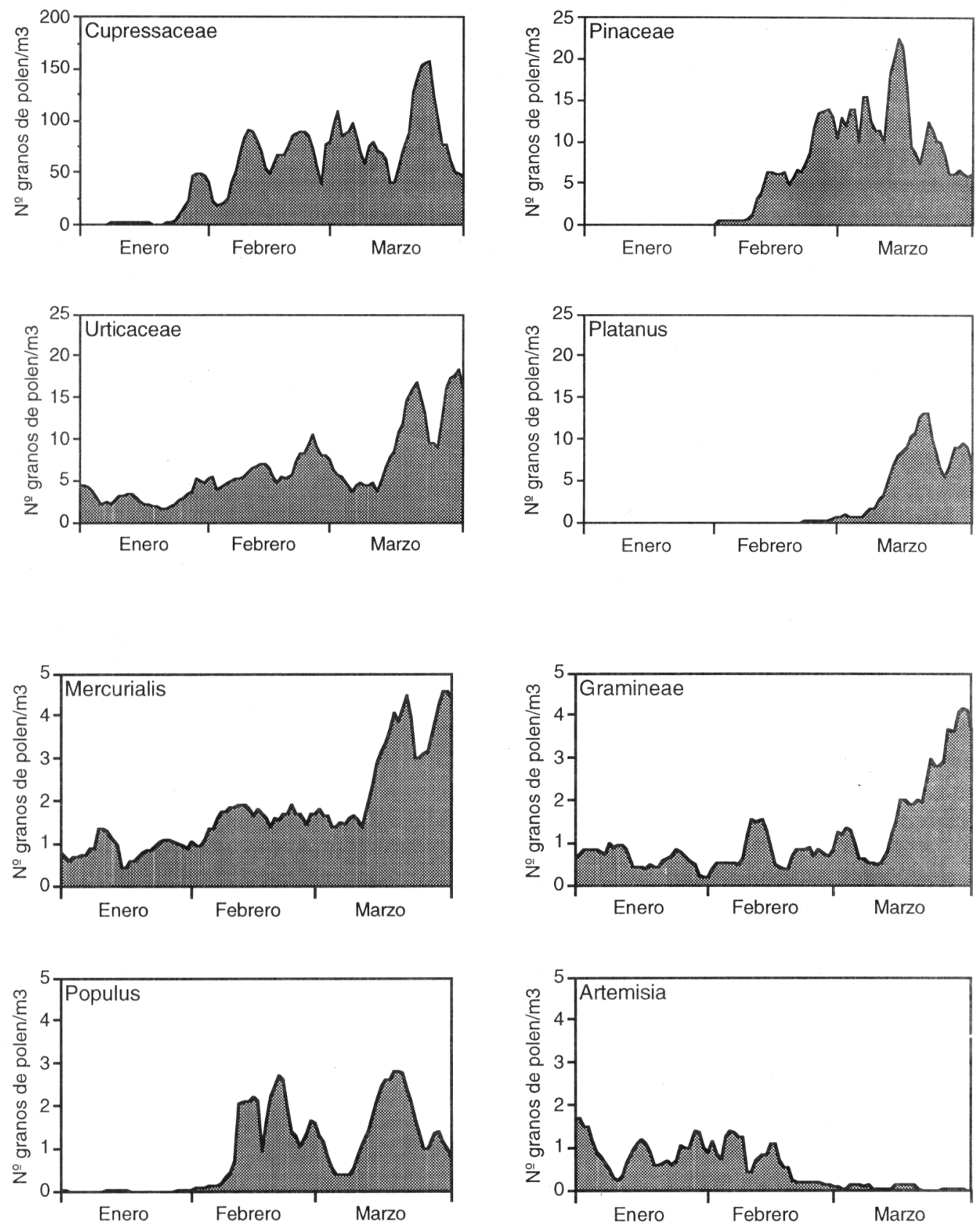

Figura 1. Evolución de las concentraciones atmosféricas de polen de los 12 taxones estudiados durante el periodo invernal en Málaga. Airborne pollen concentration trends of 12 taxa studied during winter in Malaga. 

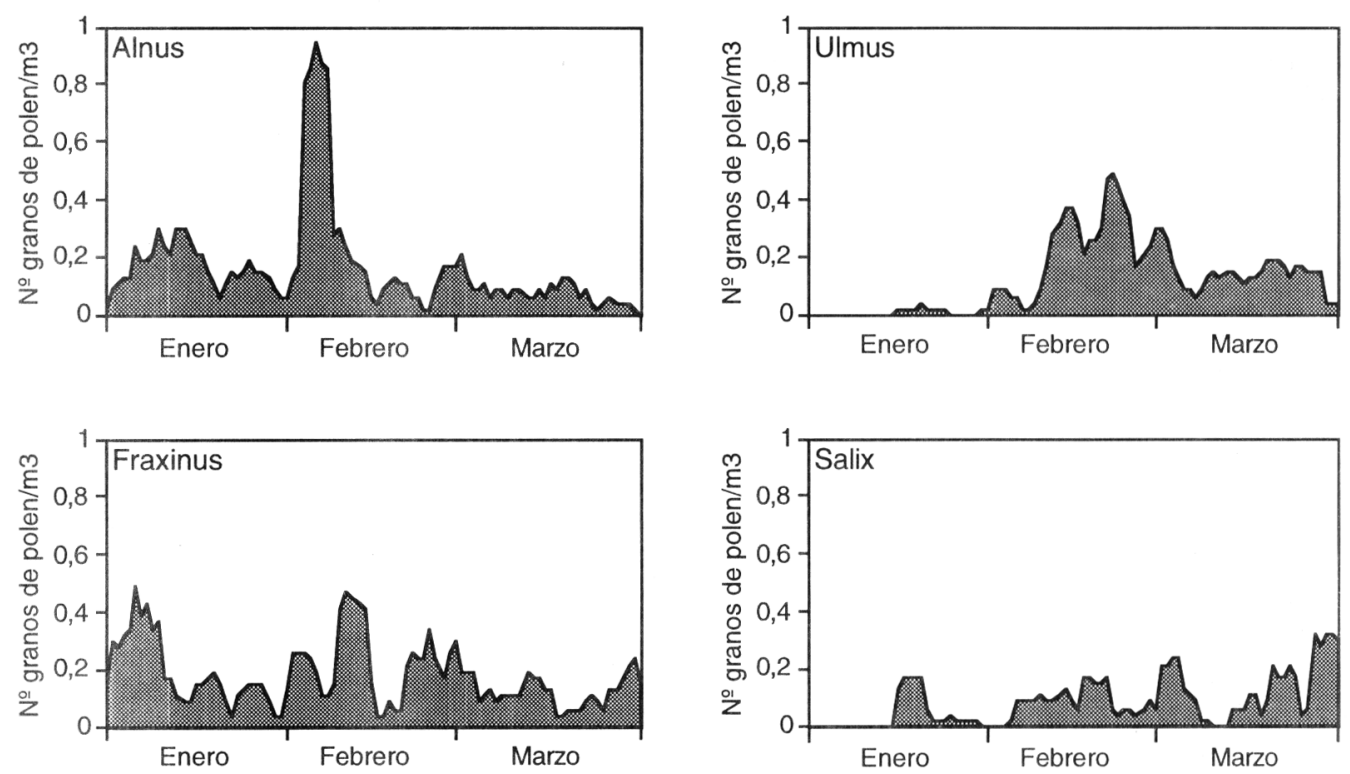

Figura 1 (continuación). Evolución de las concentraciones atmosféricas de polen de los 12 taxones estudiados durante el periodo invernal en Málaga. Airborne pollen concentration trends of 12 taxa studied during winter in Malaga

polen detectadas permanecen muy bajas, siendo uno de los meses con concentraciones más bajas de todo el año (Cabezudo et al., 1994; Recio et al. 1995; Toro et al., 1996). A finales de Enero o principios de Febrero las concentraciones totales se incrementan considerablemente, debido al aumento de polen de Cupressaceae fundamentalmente, siendo éste el tipo polínico predominante durante el mes de Febrero. Durante el mes de Marzo vuelve a producirse un nuevo pico, de menor intensidad, debido a que se inicia la floración de Platanus y comienzan a aparecer algunos taxones primaverales.

En la tabla 2 se observa que la mayoría de los taxones están muy bien representados durante el invierno, siendo escasos durante el resto del año. Sin embargo, en el caso de las Gramíneas, durante el invierno sólo se recoge un $5,81 \%$ del total anual, ya que habitualmente tienen su pico máximo durante los meses de
Mayo y Junio (tab. 3). Algo similar sucede con Artemisia, cuyo período de polinación se produce mayoritariamente en Diciembre.

En general, la mayoría de los taxones

\begin{tabular}{llc}
\hline Taxón & Total invierno & \% invierno \\
\hline Cupressaceae & 3382 & 82,90 \\
Pinaceae & 515 & 58,19 \\
Urticaceae & 468 & 40,83 \\
Platanus & 200 & 87,12 \\
Mercurialis & 149 & 68,27 \\
Gramineae & 85 & 5,81 \\
Populus & 81 & 89,72 \\
Artemisia & 58 & 25,25 \\
Fraxinus & 18 & 43,53 \\
Ulmus & 12 & 95,85 \\
Alnus & 10 & 90,19 \\
Salix & 7 & 83,31 \\
\hline
\end{tabular}

Tabla 2. Valores totales y porcentajes (con respecto al total anual) alcanzados durante el invierno por los 12 taxones estudiados (media de 5 años). Total and percentage values (out of total annual) registered by the twelve taxa studied during winter (5 years average). 
estudiados obtuvieron sus días picos en los meses de invierno (tab. 3), excepto los anteriormente citados, Gramineae y Artemisia, que los obtienen en primavera y otoño, respectivamente, y Urticaceae, que a veces lo obtiene en Abril.

Aún habiéndose realizado medias móviles de cinco días y media de los registros de cinco años, se observan importantes fluctuaciones en las curvas de distribución del polen (fig. 1). Estas fluctuaciones pueden ser debidas fundamentalmente a dos motivos. En los taxones bien representados, debido a que el periodo en que se producen los valores máximos de cada año no resulta coincidente. Esto sucede en Cupresaceae, que normalmente alcanza sus concentraciones máximas en Febrero, pero que en 1994 tuvo un fuerte pico durante la última semana del mes de Enero (Recio et al., 1995; Cabezudo et al., 1996), y en 1995 el pico máximo se retrasó a la primera semana de Abril (Toro et al., 1996). El segundo motivo

\begin{tabular}{|c|c|c|c|c|c|}
\hline Taxón & 1992 & 1993 & 1994 & 1995 & 1996 \\
\hline Artemisia & $\begin{array}{c}50 \\
(22 \mathrm{Dic} .)\end{array}$ & $\begin{array}{c}17 \\
\text { (12 Dic.) }\end{array}$ & $\stackrel{8}{8}$ (2 Dic., 3 Dic.) & $\begin{array}{c}16 \\
\text { (28 Ago., } 11 \text { Nov.) }\end{array}$ & - \\
\hline Alnus & $\begin{array}{c}1 \\
\text { (Varios) }\end{array}$ & $\begin{array}{c}1 \\
\text { (Varios) }\end{array}$ & $\begin{array}{c}3 \\
\text { (28 Dic.) }\end{array}$ & $\begin{array}{c}1 \\
\text { (Varios) }\end{array}$ & $\begin{array}{c}16 \\
(5 \text { Feb.) }\end{array}$ \\
\hline Populus & $\begin{array}{c}29 \\
(13 \text { Feb.) }\end{array}$ & $\begin{array}{l}5 \\
\text { (21 Mar.) }\end{array}$ & $\begin{array}{c}10 \\
\text { (20 Mar.) }\end{array}$ & (9 Abr.) & $\begin{array}{c}17 \\
(19 \text { Feb. })\end{array}$ \\
\hline Ulmus & $\begin{array}{l}2 \\
(21 \mathrm{Feb} .)\end{array}$ & $\stackrel{1}{1}$ (28 Feb., 23 Mar.) & (3 Feb., 19 Feb.) & (22 Feb.) & $\begin{array}{l}2 \\
\text { (26 Mar.) }\end{array}$ \\
\hline Cupressaceae & $\begin{array}{c}435 \\
(17 \text { Feb. })\end{array}$ & $\begin{array}{c}414 \\
(22 \text { Feb.) }\end{array}$ & $\begin{array}{c}602 \\
\text { (29 Ene.) }\end{array}$ & $\begin{array}{c}157 \\
(4 \mathrm{Abr} .)\end{array}$ & $\begin{array}{c}1072 \\
(29 \mathrm{Feb} .)\end{array}$ \\
\hline Pinaceae & $\begin{array}{c}82 \\
(23 \text { Mar.) }\end{array}$ & $\begin{array}{c}176 \\
(8 \text { Mar.) }\end{array}$ & $\begin{array}{c}59 \\
(14 \text { Feb.) }\end{array}$ & $\begin{array}{c}116 \\
\text { (13 Mar.) }\end{array}$ & $\begin{array}{c}164 \\
\text { (14 Mar.) }\end{array}$ \\
\hline Platanus & $\begin{array}{c}59 \\
\text { (14 Mar.) }\end{array}$ & $\begin{array}{c}43 \\
\text { (21 Mar.) }\end{array}$ & $\begin{array}{c}17 \\
\text { (19 Mar.) }\end{array}$ & $\begin{array}{c}53 \\
\text { (19 Mar.) }\end{array}$ & $\begin{array}{c}59 \\
\text { (29 Mar.) }\end{array}$ \\
\hline Mercurialis & $\begin{array}{c}7 \\
\text { (23 Mar.) }\end{array}$ & $\begin{array}{c}12 \\
(4 \mathrm{Abr} .)\end{array}$ & $\begin{array}{c}7 \\
\text { (1 Mar.) }\end{array}$ & $\begin{array}{c}10 \\
\text { (19 Mar.) }\end{array}$ & $\begin{array}{c}16 \\
\text { (29 Mar.) }\end{array}$ \\
\hline Salix & $\begin{array}{l}4 \\
\text { (29 Mar.) }\end{array}$ & $\stackrel{1}{1}$ & $\stackrel{3}{\text { (18 Ene.) }}$ & $\stackrel{1}{1}$ & $\begin{array}{c}3 \\
(2 \text { Mar. })\end{array}$ \\
\hline Urticaceae & $\begin{array}{c}17 \\
\text { (18 Mar.) }\end{array}$ & $\begin{array}{c}29 \\
(7 \mathrm{Abr} .)\end{array}$ & $\begin{array}{c}47 \\
\text { (21 Mar.) }\end{array}$ & $\begin{array}{c}46 \\
\text { (18 Mar.) }\end{array}$ & $\begin{array}{c}66 \\
(14 \mathrm{Abr} .)\end{array}$ \\
\hline Fraxinus & (20 $\stackrel{3}{\text { Dic. })}$ & $\stackrel{3}{3}$ (22 Feb.) & (8 Ene., 11 Feb.) & $\begin{array}{c}24 \\
(15 \mathrm{Abr} .)\end{array}$ & $\begin{array}{c}2 \\
\text { (Varios) }\end{array}$ \\
\hline Gramineae & $\begin{array}{c}60 \\
\text { (11 May.) }\end{array}$ & $\begin{array}{c}99 \\
\text { (2 Jun.) }\end{array}$ & $\begin{array}{c}149 \\
\text { (8 May.) }\end{array}$ & $\begin{array}{c}67 \\
\text { (25 May.) }\end{array}$ & $\begin{array}{c}487 \\
\text { (2 Jun.) }\end{array}$ \\
\hline
\end{tabular}

Tabla 3. Máximos diarios (valor medio diario) y fecha de los días pico obtenidos en cada año de muestreo por los doce taxones estudiados. Daily maximum (daily average value) and the date of peak days obtained by 12 taxa studied in each year sampled. 
estaría en la influencia de la dirección del viento en aquellos taxones que habitan en zonas alejadas del punto de muestreo, como artemisias, chopos, álamos, olmos, fresnos y sauces. En Málaga las artemisias se encuentran únicamente formando poblaciones densas en la zona oriental de la provincia y en su límite con la provincia de Granada, por lo que para que sus pólenes sean detectados por nuestro muestrador es necesario que soplen vientos fuertes de levante (E). Por el contrario, los árboles citados anteriormente habitan en riberas umbrosas, dispersas y escasamente representadas en la provincia de Málaga, encontrándose sólo de forma abundante en la zona occidental y en su límite con la provincia de Cádiz, por lo que sólo se detectan concentraciones significativas cuando los vientos soplan de poniente $(\mathrm{O})$. Se ha observado que los días de registro de estos pólenes coincidieron con fuertes vientos de dirección favorable para sean arrastrados desde la zona donde abundan hasta Málaga.

AGRADECIMIENTOS. Los autores desean agradecer a las Consejerías de Salud y Educación de la Junta de Andalucía la colaboración prestada a la Red Andaluza de Aerobiología, para el desarrollo de este trabajo.

\section{BIBLIOGRAFÍA}

CABEZUDO, B., M.M. TRIGO, M. RECIO y F.J. TORO -1994- Contenido polínico de la atmósfera de Málaga: Años 1992 y 1993. Acta Bot. Malacitana, 19: 137-144.

CABEZUDO, B., M. RECIO, M.M. TRIGO, y F.J. TORO - 1996- Aerobiología de la Costa del Sol. Málaga (1992-94). Boletín REA, 2. (En prensa). DOMÍNGUEZ VILCHES, E., C. GALÁN SOLDEVILLA, F. VILLAMANDOS DE LA TORRE \& F. INFANTE GARCÍAPANTALEÓN - 1991- Handling and evaluation of the data from the aerobiological sampling. Monografías REA/EAN, 1: 1-18.
NILSSON, S. \& F.TH.M. SPIEKSMA, (eds.)-1992Traveller's Allergy Service Guide. Swedish Museum of Natural History and Fisons Sweden $\mathrm{AB}$, Sweden.

RECIO, M., M.M. TRIGO, F.J. TORO y B CABEZUDO -1995- Contenido polínico de la atmósfera de Málaga: Año 1994. Acta Bot. Malacitana, 20: 83-90.

SPIEKSMA, F.TH.M. -1991- Regional European Pollen Calendars. In: G. D’AMATO, F.TH.M SPIEKSMA \& S. BONINI (eds.) Allergenic pollen and pollinosis in Europe, pp. 49-65. Blackwell Scientific Publications, Oxford.

SPIEKSMA, F.TH.M. \& G. FRENGUELLI -1991Allergenic significance of Alnus (Alder) pollen In: G. D'AMATO, F.TH.M. SPIEKSMA \& S BONINI (eds.) Allergenic pollen and pollinosis in Europe, pp. 85-86. Blackwell Scientific Publications, Oxford.

TORO, F.J., M. RECIO, M.M. TRIGO y B. CABEZUDO - 1996-. Contenido polínico de la atmósfera de Málaga: Año 1995. Acta Bot. Malacitana, 21: 57-63.

Aceptado para su publicación en Mayo de 1996

Dirección de los autores. Departamento de Biología Vegetal. Facultad de Ciencias. Universidad de Málaga. Apdo. 59. 29080, Málaga. Fax. (95) 2131944 